\title{
Advances in anatomical bases for head and neck surgery
}

\author{
F. Crampon ${ }^{1,2} \cdot$ F. Duparc ${ }^{1} \cdot$ B. Grignon ${ }^{3} \cdot$ O. Trost $^{1,4}$
}

Published online: 12 May 2018

(c) Springer-Verlag France SAS, part of Springer Nature 2018

This new issue of Surgical and Radiologic Anatomy is consecrated to head and neck anatomy and surgery. Here are proposed some original studies and relevant anatomical variations related to oral, maxillofacial, and ENT surgery.

The first section of this special issue comprises original works useful in dental implants procedures and in orthognathic surgery. Indeed the knowledge of precise course of the mandibular canal, from the mandibular foramen to the mental foramen, and its variations (accessory foramens, retromandibular canal, coronoid foramen ...) is mandatory to perform dental implants or pre-implant surgery in maximal safety conditions. Likewise, mandibular sagittal osteotomies are being more and more commonly performed and require optimal knowledge of intramandibular structures to improve the prognosis for sequential disorders of lower lip and chin sensitivity. The use of CBCT (cone beam) in these studies is perfectly in tune with the times and corresponds to the modernity of clinical practice.

Maxillofacial disharmonies result from growth troubles, due to bone abnormalities and/or orofacial dysfunctions. Two original studies of the perinatal mandibular growth patterns and of the intrinsic and extrinsic organizations of the

F. Duparc

fabrice.duparc@univ-rouen.fr

F. Crampon

frederic.crampon@chu-rouen.fr

B. Grignon

b.grignon@chru-nancy.fr

O. Trost

olivier.trost@chu-rouen.fr

1 Laboratory of Anatomy, Faculty of Medicine, Rouen University, 22 boulevard Gambetta, 76183 Rouen, France

2 Department of ENT Surgery, Rouen University Hospital, 1 rue de Germont, 76031 Rouen, France

3 Department of Medical Imaging, Nancy University Hospital, 29 Avenue du Maréchal de Lattre de Tassigny, 54035 Nancy, France

4 Department of Oral and Maxillofacial Surgery, Rouen University Hospital, 1 rue de Germont, 76031 Rouen, France lingual muscles will fuel discussions on this topic. Finally, an original MRI study of the pterygopalatine ganglion, a neurologic structure potentially injured in Le Fort 1 osteotomies, will conclude this topic.

The second section is dedicated to inner ear anatomy and surgery. Indeed, the treatment of perception deafness requires the installation of cochlear implants whose quality has been significantly improved for several years. Microanatomical studies lead to further evolution of these materials. A last study shows microanatomy of the cochlear aqueduct whose function remains unknown. Nevertheless, we cannot help being amazed at such beautiful images!

The various techniques of medical imaging that have been used for these anatomical studies reflect the so much important place of the depiction of anatomy through radiography, microcomputed tomodensitometry, 3D-computed tomodensitometry, magnetic resonance angiography. These performant techniques leave also place to the anatomical examination.

In 2010, a special issue contained publications "Around the mandible". Eight years after, these topics remain of importance, this field of research is still very active, and we hope anatomists and clinicians will appreciate these advances. 